DEMONSTRATIO MATHEMATICA

Vol. XLIII No 2010

\title{
Tadashi Ashikaga
}

\section{LOCAL SIGNATURE OF FIBERED COMPLEX SURFACES VIA MODULI AND MONODROMY}

\begin{abstract}
The signature of fibered complex surfaces is sometimes localized at finite fiber germs. We analyze this phenomenon by using datum of local monodromy in the mapping class group and a certain rational divisor on the moduli space of stable curves.
\end{abstract}

\section{Introduction}

Let $f: S \rightarrow B$ be a proper surjective holomorphic map from a compact complex surface $S$ to a Riemann surface $B$ such that the general fiber of $f$ is a Riemann surface of genus $g$. Let Sign $S$ be the signature of the intersection form on $H^{2}(S, \mathbf{Q})$. If there exist a finite number of fiber germs $\left(f, F_{i}\right), F_{i}=f^{-1}\left(P_{i}\right)\left(1 \leq i \leq s, P_{i} \in B\right)$ and a local invariant $\sigma\left(f, F_{i}\right)$ is geometrically well-defined such that

$$
\operatorname{Sign} S=\sum_{i=1}^{s} \sigma\left(f, F_{i}\right),
$$

then we say Sign $S$ is localized and call $\sigma\left(f, F_{i}\right)$ a local signature. Our problem here is to discuss how to formulate and also how to calculate the local signature. It should be formulated by depending on the nature of the general fiber of $f$ (see $\S 2.2$ ).

One of the motivation of this problem comes from the study of the topological structure of Lefschetz fibration, which also relates to symplectic geometry. The other motivation comes from the algebro-geometric study of complex surfaces, especially geography of surfaces of general type.

The author already published two survey papers around these fields with Konno [5] and with Endo [3] respectively. In very recent years after that,

2010 Mathematics Subject Classification: Primary: 14D06, 14H10, 32Q55, 55R55, 57M50; Secondary: 14J29, 14J80, 32S40, 57R18, 57R45.

Key words and phrases: signature, degeneration, monodromy, Riemann surface, complex surface, localization, moduli of curves. 
new methods and new applications furthermore appeared. If we restrict our attention only to the direct formulation of local signature, they are totally the followings in author's knowledge:

(1) In the case where $f$ is an elliptic fibration, Matsumoto [24] formulated and calculated it by using Meyer function. Atiyah [7] essentially described it by the method of "signature defect" analogous to the Hirzebruch's signature defect of isolated singularities in some sense (see Remark 1.3.6).

(2) Assume $f$ is a genus 2 fibration. Horikawa [16] essentially calculated it by the method of double covering. Ueno [35] formulated and calculated it by using even theta constant. Matsumoto [25] formulated and calculated it by the method of Meyer function. Iida [17] formulated and calculated it by using the adiabatic limit of eta invariant.

(3) Assume $f$ is a hyperelliptic fibration of arbitrary genus. Endo [11] and Morifuji [26] formulated and calculated it for Lefschetz fiber germ by using Meyer function. Xiao [36] formulated it by using the method of double covering. See also Arakawa and the author [1].

From (1) to (3), all the germs with non-zero local signatures are germs of singular fibers. Although from (4) below, "a certain special" smooth fibers also carry non-zero local signatures, which seems to be a remarkable property of this notion.

(4) Assume $f$ is a non-hyperelliptic fibration of genus 3. Reid [30] essentially formulated it by using the relative canonical algebra. Kuno [21] formulated and calculated it for Lefschetz fiber germs by using the method of Meyer function. (Kuno also formulated it to a fibration of plane curves.) Chen and Tan [9] essentially formulated it by using the method of triple coverings.

(5) Konno [19] essentially formulated it for fibrations of arbitrary genus whose general fiber has a preassigned Clifford index by using the relative canonical algebra. If $f$ is a Clifford general fibration of odd genus, his argument is more precise than the general case. For a Clifford general fibration of odd genus (which is called Harris-Mumford general fibration in this note), Yoshikawa and the author [6] formulated and calculated it in some sense by using the Harris-Mumford formula and the local signature defect of the author [2].

(6) In the case where $f$ is a genus 4 fibration whose general fiber has two mutually distinct trigonal structures, Yoshikawa and the author [6] formulated it by using Eisenbud-Harris formula. Konno [20] formulated it by using the relative canonical algebra. 
In the above references, we include the results which are concerned with the notion of Horikawa index, because Horikawa index is a "precise version" of local signature in some sense (cf. $[5, \S 2]$ ).

Although the following (7) and (8) are reports of certain symposiums written in Japanese at this stage, we add them here for their value.

(7) Yoshikawa [37] formulated and calculated it for Lefschetz fiber germs for a certain generic fibration by using even theta constant.

(8) Furuta [12], [13] presented a method of formulation for a certain generic fibration by using eta invariant and odd theta constant. Sato [31] realized it for a certain special fibration.

In Polish-Japanese Singularity Conference at Sopot (2007), we talked about a new type of local signature exploited in [2] and [6]. Although [2] and [6] are not yet under publication at this stage (2008 August), the author would like to announce here the main result of them. For the details, see [2], [6].

To say philosophically, our notion of local signature discussed here consists of two terms of "moduli" and "monodromy" :

$$
\text { Local signature }=\text { "Moduli" term + "Monodromy" term. }
$$

If $f$ is stable, only the moduli term contribute to the local signature. If $f$ is unstable, the monodromy term appear as a correction term between the unstable fiber germ and the germ of its stable reduction. We discuss the monodromy term in $\S 1$. By combining the moduli term with it, we present our local signature in $\S 2$.

The author would like to thanks the referee for useful comments.

\section{Local signature defect}

This section is a summary of the main result of [2]. We define the local signature defect of a degeneration of Riemann surfaces, and express it explicitly in terms of the data of topological monodromy.

1.1. Let $0<\epsilon<1$ be a sufficiently small real number. Let $f: S \rightarrow \Delta$ a normally minimal degeneration of genus $g$ over a closed $\epsilon$-disk $\Delta=\{t \in$ $\mathrm{C}|| t \mid \leq \epsilon\}$. By definition, $S$ is a smooth complex surface with boundary, the general fiber $f^{-1}(t)(t \neq 0)$ is a closed Riemann surface of genus $g$ and the reduced scheme $F_{\text {red }}$ of the central fiber $F=f^{-1}(0)$ is normal crossing and any $(-1)$ curve in the components of $F$ meet other components of $F$ at least three points. We denote the restriction of $f$ to its boundary by $\partial f: \partial S \longrightarrow \partial \Delta \simeq S^{1}$.

Since the restricted family over the punctured disk $f^{-1}\left(\Delta^{*}\right) \rightarrow \Delta^{*}=$ $\Delta \backslash\{0\}$ is differentiably locally trivial, there exists a Riemannian metric 
$h_{S}=\left\{h_{i j}\right\}$ on $S$ such that the restriction over a tubular neighborhhod of $\partial S$ is a product metric. We put $h_{\partial S}$ the restriction of $h_{S}$ to $\partial S$. The choice of $h_{S}$ is not unique, and we fix one of them. Let $\operatorname{Sign} S$ be the signature of the intersection form on $H^{2}(S, \partial S ; \mathbf{Q})$, and $\eta\left(\partial S, h_{\partial S}\right)$ be the eta-invariant of Atiyah-Patodi-Singer [8]. Inspired by Furuta's discussion [12 ${ }^{1}$, we put

$$
\sigma\left(f, F ; h_{\partial S}\right)=\operatorname{Sign} S+\eta\left(\partial S, h_{\partial S}\right) .
$$

Let $\tilde{f}: \widetilde{S} \rightarrow \widetilde{\Delta}$ be the stable reduction of degree $N$ of $f$, and let $\rho$ : $\widetilde{\Delta} \rightarrow \Delta, \widetilde{\rho}: \widetilde{S} \rightarrow S$ be the natural maps. By definition, $\rho$ is a cyclic cover of degree $N$ totally ramified at the origin, $\widetilde{S}$ is a normal 2-dimensional complex space with at most rational double points of type $A$ which is birationally equivalent to the fiber product of $S$ and $\widetilde{\Delta}$ over $\Delta$, and the central fiber $\widetilde{F}=\widetilde{f}^{-1}(0)$ is a stable curve in the sense of [10]. Namely, $\widetilde{F}$ is reduced, normal crossing and has no $(-2)$ component.

Let $h_{\widetilde{S}}$ be a Riemannian metric on $\widetilde{S}$ so that $h_{\widetilde{S}}$ is an extension of the natural pull back of the restricted metric of $h_{S}$ to a tubular neighborhood of $\partial S$. Since the map $\widetilde{\rho}$ is umramified near the boundary, it is well-defined. The metric $h_{\widetilde{S}}$ is a product metric near the boundary $\partial \widetilde{S}$, and the eta-invariant $\eta\left(\partial \widetilde{S}, h_{\partial \widetilde{S}}\right)$ is well-defined. We also put $\sigma\left(\widetilde{f}, \widetilde{F} ; h_{\partial \widetilde{S}}\right)=\operatorname{Sign}(\widetilde{S})+\eta\left(\partial \widetilde{S}, h_{\partial \widetilde{S}}\right)$.

Definition 1.1.1. The local signature defect of the fiber germ $(f, F)$ of order $N$ is defined as

$$
\operatorname{Lsd}(f, F ; N)=\sigma\left(f, F ; h_{\partial S}\right)-\frac{1}{N} \sigma\left(\tilde{f}, \widetilde{F} ; h_{\partial \widetilde{S}}\right) .
$$

Since the difference $\eta\left(\partial S, h_{S}\right)-(1 / N) \eta\left(\partial \widetilde{S}, h_{\partial \widetilde{S}}\right)$ is independent of the choice of the metric $h_{\partial S}$ by [8, II, Th. 2.4], so is $\operatorname{Lsd}(f, F ; N)$.

1.2. Let $\mu=\mu_{f}: \Sigma_{g} \rightarrow \Sigma_{g}$ be the monodormy map of $f\left(\Sigma_{g}\right.$ is a closed Riemann surface of genus $g$ ). It is well-known that $\mu$ is a pseudo-periodic map of negative twist as an element of the mapping class group $\boldsymbol{\Gamma}_{g}$ of genus $g$ (cf. $[5, \S 3])$. Note that $\mu$ is well-defined as a representative of a conjugacy class of $\boldsymbol{\Gamma}_{g}$. Let

$$
\Sigma_{g}=\mathbf{A} \cup \mathbf{B}
$$

be the decomposition into the annulus-part $\mathbf{A}=\amalg \mathcal{A}_{j}$ which is the disjoint union of the annular neighborhoods $\mathcal{A}_{j}$ of simple closed curves on $\Sigma_{g}$ belonging to the admissible system of cut curves, and the body-part $\mathbf{B}=\coprod B_{i}$ which is the disjoint union of Riemann surfaces $B_{i}$ with boundary, such that the boundary set $\partial \mathbf{B}$ coincides with the boundary set $\partial \mathbf{A}$. The restriction $\left.\mu\right|_{\mathbf{B}}$ is periodic, i.e. the power $\left(\left.\mu\right|_{\mathbf{B}}\right)^{N}$ for some natural number $N$ is the

\footnotetext{
${ }^{1}$ Furuta [12], [13] discussed this type of invariants under a more general setting of the linear connection.
} 
identity map id $\mathbf{B}_{\mathbf{B}}$. The power of the restriction $\left(\left.\mu\right|_{\mathcal{A}_{j}}\right)^{N}$ to each annulus $\mathcal{A}_{j}$ is a right-handed integral Dehn twist. We call such $N$ a pseudo-period of $\mu$. Note that $N$ is a multiple of the minimal pseudo-period $N_{0}$ (i.e. the minimal natural number among all the pseudo-periods).

Note that, since the stable reduction is possible if and only if the covering degree of the base change coincides with the pseudo-period of the monodormy map, we use the same symbol $N$ (cf. [2, §2], [5, §3]).

Now let $[\mu]$ be the equivalence class of $\mu$ of the set $\widehat{\mathcal{M}}_{g}$ of the conjugacy class of the mapping class group of genus $g$. We review the conjugacy invariants of $[\mu]$ introduced by Nielsen [28] .

Let $\vec{C}$ be an oriented simple closed curve on $\Sigma_{g}$. Suppose there is a natural number $m=m(\vec{C})$ such that $\mu^{m}(\vec{C})=\vec{C}$ as a set, where $m$ is assumed to be the minimal number which enjoys this property. Moreover suppose $(\mu \mid \vec{C})^{m}$ is periodic of order $\lambda=\lambda(\vec{C}) \geq 1$. Then for any point $R$ on $\vec{C}$, there is a natural number $\sigma=\sigma(\vec{C})$ with $1 \leq \sigma \leq \lambda-1$ such that the iteration of $\mu^{m}$ are situated in the order $\left(R, \mu^{m \sigma}(R), \mu^{2 m \sigma}(R), \cdots, \mu^{(\lambda-1) m \sigma}(R)\right)$ when viewed in the direction of $\vec{C}$. The set of triple $(m(\vec{C}), \lambda(\vec{C}), \sigma(\vec{C}))$ is called Nielen's valency at $\vec{C}([27])$. Let $\delta=\delta(\vec{C})$ be the integer which satisfies $\sigma \delta \equiv 1(\bmod \lambda)$ and $1 \leq \delta \leq \lambda-1$. Then the map $\left.\mu^{m}\right|_{\vec{C}}$ behaves as the rotation of angle $2 \pi \delta / \lambda$ in a suitable parametrization of $\vec{C}$. Since the number $\delta$ is also important in our argument, we simply call the quardruplet $(m(\vec{C}), \lambda(\vec{C}), \sigma(\vec{C}), \delta(\vec{C}))$ the valency at $\vec{C}$.

Let $Q$ be a point on the inside of a body component $B_{i} \backslash \partial B_{i}$. Let $m\left(B_{i}\right)$ be the minimal natural number such that $\left.\mu^{m\left(B_{i}\right)}\right|_{B_{i}}=\mathrm{id}_{B_{i}}$. If there exists a natural number $m=m(Q)$ which is strictly smaller than $m\left(B_{i}\right)$ such that the points $\left\{Q, \mu(Q), \cdots, \mu^{m-1}(Q)\right\}$ are distinct each other and $\mu^{m}(Q)=Q$, we call $Q$ a multiple point. Then there exists a disk neighborhood $D_{Q}$ of $Q$ which is invariant under the action $\mu^{m}$. The valency $(m(Q), \lambda(Q), \sigma(Q), \delta(Q))$ at $Q$ is defined to be the valency of the boundary curve $\partial D_{Q}$ of $D_{Q}$ whose orientation is defined from the outside of the disk. (Note that the orientation of $\partial D_{Q}$ is defined from the inside in [22].)

For an annulus component $\mathcal{A}_{j}$, we put $\partial \mathcal{A}_{j}=\partial \mathcal{A}_{j}^{(1)} \amalg \partial \mathcal{A}_{j}^{(2)}$ the decomposition to connected components of the boundary curve where the orientation is defined here from the outside of the annulus. The valency at $\mathcal{A}_{j}$ is defined to be the couple of valencies $\left(m\left(\partial \mathcal{A}_{j}^{(k)}\right), \lambda\left(\partial \mathcal{A}_{j}^{(k)}\right), \sigma\left(\partial \mathcal{A}_{j}^{(k)}\right), \delta\left(\partial \mathcal{A}_{j}^{(k)}\right)\right)$ for $k=1,2$. If there exists a natural number $\beta$ such that $\mu^{\beta}$ interchanges the boundary components of $\mathcal{A}_{j}$, i.e. $\mu^{\beta}\left(\partial \mathcal{A}_{j}^{(1)}\right)=\partial \mathcal{A}_{j}^{(2)}$, we call $\mathcal{A}_{j}$ an amphidrome annulus. Otherwise we call $\mathcal{A}_{j}$ a non-amphidrome annulus.

Let $\alpha$ be the smallest natural number such that $\mu^{\alpha}\left(\mathcal{A}_{j}\right)=\mathcal{A}_{j}$ does not interchange the boundary components. Let $\gamma$ be a non-zero integer such that 
$\left.\mu^{\gamma}\right|_{\partial \mathcal{A}_{j}}$ is the identity map. Then $\gamma$ is a multiple of $\alpha$, and $\mu^{\gamma}: \mathcal{A}_{j} \rightarrow \mathcal{A}_{j}$ is a result of $e$ full Dehn twist, $e$ being an integer. Then we define the screw number at $\mathcal{A}_{j}$ by $\mathbf{s}\left(\mathcal{A}_{j}\right):=e \alpha / \gamma$.

The theorem of Nielsen [28] and Matsumoto-Montesinos [22] says that the conjugacy class of $\mu$ is determined by the data of

(i) valencies at multiple points $\{Q\}$ and the annuli $\left\{\mathcal{A}_{j}\right\}$,

(ii) screw numbers at the annuli $\left\{\mathcal{A}_{j}\right\}$,

(iii) the action of $\mu$ to the extended partition graph $\boldsymbol{\Gamma}(\mu)$, i.e., the onedimensional oriented graph whose points correspond to $\left\{B_{i}\right\}$ and whose segments correspond to $\left\{\mathcal{A}_{j}\right\}$ in a natural way.

1.3. By depending on the discussion in $\S 1.2$, the invariants of the orbits of the monodormy map are naturally defined as follows. Let $\Sigma_{g}=\mathbf{B} \cup \mathbf{A}^{\prime} \cup \mathbf{A}^{\prime \prime}$ be the decomposition to the set of body connected components $\mathbf{B}=\coprod B_{i}$, the set of non-amphidrome annuli $\mathbf{A}^{\prime}=\amalg \mathcal{A}_{j}$ and the set of amphidrome annuli $\mathbf{A}^{\prime \prime}=\coprod \widetilde{\mathcal{A}}_{k}$ with respect to (the representative of) the monodromy map $\mu_{f}: \Sigma_{g} \rightarrow \Sigma_{g}$. Let $\mathbf{B} / \sim=\amalg\left[B_{i}\right]$ be the orbit decomposition with respect to the cyclic action generated by $\mu_{f}$. Namely two components $B_{i_{1}}$ and $B_{i_{2}}$ are equivalent iff $B_{i_{2}}=\left(\mu_{f}\right)^{n}\left(B_{i_{1}}\right)$ for some integer $n$, and $\mathbf{B} / \sim$ is its equivalence class. We set $\mathbf{A}^{\prime} / \sim=\amalg\left[\mathcal{A}_{j}\right], \mathbf{A}^{\prime \prime} / \sim=\amalg\left[\widetilde{\mathcal{A}}_{k}\right]$ similarly.

Let $\left\{m_{\alpha}, \lambda_{\alpha}^{(i)}, \sigma_{\alpha}^{(i)}, \delta_{\alpha}^{(i)}\right\}_{1 \leq \alpha \leq \varphi(i)}$ be the set of all the valencies which are attached to multiple points and boundary curves of $B_{i}$. Let

$$
\begin{aligned}
\frac{\lambda_{\alpha}^{(i)}}{\sigma_{\alpha}^{(i)}} & =K_{1}(\alpha, i)-\frac{1}{K_{2}(\alpha, i)-\frac{1}{K_{3}(\alpha, i)-\cdots \frac{1}{K_{r}(\alpha, i)}}} \\
& :=\left[\left[K_{1}(\alpha, i), K_{2}(\alpha, i), \cdots, K_{r}(\alpha, i)\right]\right]
\end{aligned}
$$

be the continued linear fraction. The sequence $\left\{n_{k}\right\}_{k=0}^{r}$ of natural numbers which satisfies $n_{0}=\lambda_{\alpha}^{(i)}, n_{1}=\sigma_{\alpha}^{(i)}$ and

$$
n_{k}=K_{k-1}(\alpha, i) \cdot n_{k-1}-n_{k-2}(2 \leq k \leq r+1)
$$

is called the multiplicity sequence of the above continued linear fraction.

Let $\left(m\left(\mathcal{A}_{j}\right), \lambda^{(1)}\left(\mathcal{A}_{j}\right), \sigma^{(1)}\left(\mathcal{A}_{j}\right), \delta^{(1)}\left(\mathcal{A}_{j}\right)\right)$ and $\left(m\left(\mathcal{A}_{j}\right), \lambda^{(2)}\left(\mathcal{A}_{j}\right), \sigma^{(2)}\left(\mathcal{A}_{j}\right)\right.$, $\left.\delta^{(2)}\left(\mathcal{A}_{j}\right)\right)$ be the valencies of both boundary curves of the non-amphidrome annulus $\mathcal{A}_{j}$. (We write $\lambda^{(1)}\left(\mathcal{A}_{j}\right)=\lambda^{(1)}$ etc. for simplicity if it is no confusion.) The screw number is written as

$$
\mathbf{s}\left(\mathcal{A}_{j}\right)=-\frac{\delta^{(1)}}{\lambda^{(1)}}-\frac{\delta^{(2)}}{\lambda^{(2)}}-\mathbf{K}\left(\mathcal{A}_{j}\right)
$$


where $\mathbf{K}\left(\mathcal{A}_{j}\right)$ is an integer greater than or equal to $-1([22])$. Set

$$
\frac{\lambda^{(1)}}{\sigma^{(1)}}=\left[\left[K_{1}^{(j)}, K_{2}^{(j)}, \cdots, K_{r(j)}^{(j)}\right]\right], \quad \frac{\lambda^{(2)}}{\sigma^{(2)}}=\left[\left[L_{1}^{(j)}, L_{2}^{(j)}, \cdots, L_{r^{\prime}(j)}^{(j)}\right]\right],
$$

and let $\left\{n_{i}\right\}_{i=0}^{r+1},\left\{m_{i}\right\}_{i=0}^{r^{\prime}+1}$ be their multiplicity sequences of $\lambda^{(1)} / \sigma^{(1)}$ and $\lambda^{(2)} / \sigma^{(2)}$ respectively. Moreover we put

$$
c\left(\mathcal{A}_{j}\right)=\operatorname{gcd}\left(\lambda^{(1)}, \lambda^{(2)}\right), \quad \ell\left(\mathcal{A}_{j}\right)=\frac{N}{\operatorname{lcm}\left(\lambda^{(1)}, \lambda^{(2)}\right) \cdot m\left(\mathcal{A}_{j}\right)} .
$$

Assume $\mathbf{K}\left(\mathcal{A}_{j}\right)=-1$. There exists an unique pair $\left(\omega(j), \omega^{\prime}(j)\right)$ of integers with $0 \leq \omega(j) \leq r(j), 0 \leq \omega^{\prime}(j) \leq r^{\prime}(j)$ which satisfies

$$
n_{\omega(j)}=m_{\omega^{\prime}(j)}, \quad n_{\omega(j)+1}+m_{\omega^{\prime}(j)+1}=n_{\omega(j)},
$$

from $[22, \S 6]$ and $[33, \S 6]$. Assume $\omega(j) \geq 1, \omega^{\prime}(j) \geq 1$. Let $\hat{\sigma}^{(2)}$ be the natural number which satisfies $\sigma^{(2)} \delta^{(2)}=\widehat{\sigma}^{(2)} \lambda^{(2)}+1$. According to Takamura [33], we define integers $d, v^{*}$ by

$$
d=-\lambda^{(1)} \lambda^{(2)} \mathbf{s}\left(\mathcal{A}_{j}\right), \quad v^{*}=\sigma^{(2)} \delta^{(1)}+\lambda^{(1)} \widehat{\sigma}^{(2)}-\sigma^{(1)} \lambda^{(1)} .
$$

Definition 1.3.1. A rational number $\epsilon\left(\mathcal{A}_{j}\right)$ is defined as follows:

If $\mathbf{K}\left(\mathcal{A}_{j}\right) \geq 0$, put

$$
\epsilon\left(\mathcal{A}_{j}\right)=0
$$

If $\mathbf{K}\left(\mathcal{A}_{j}\right)=-1$, put

$$
\epsilon\left(\mathcal{A}_{j}\right)= \begin{cases}\frac{1}{3}\left(\sum_{i=\omega(j)+1}^{r(j)} K_{i}^{(j)}+\sum_{i=\omega^{\prime}(j)+1}^{r^{\prime}(j)} L_{i}^{(j)}-1\right), \omega(j) \geq 1, \omega^{\prime}(j) \geq 1 \\ \frac{1}{3}\left(\sum_{i=\omega(j)+1}^{r(j)} K_{i}^{(j)}+\sum_{i=1}^{r^{\prime}(j)} L_{i}^{(j)}-\alpha^{*}-2\right), \omega(j) \geq 2, \omega^{\prime}(j)=0 \\ \frac{1}{3}\left(\sum_{i=1}^{r(j)} K_{i}^{(j)}+\sum_{i=1}^{r^{\prime}(j)} L_{i}^{(j)}-\beta^{*}-2\right), \quad \omega(j)=1, \omega^{\prime}(j)=0,\end{cases}
$$

where $\alpha^{*}$ is the integer which satisfies $1 \leq v^{*}+\alpha^{*} d \leq d-1$ and $\beta^{*}=$ $-\frac{\sigma^{(1)}}{\lambda^{(1)}}-\frac{\sigma^{(2)}}{\lambda^{(2)}}+\frac{\lambda^{(2)}}{\lambda^{(1)}}+\frac{\lambda^{(1)}}{\lambda^{(2)}}$.

Let $\left(m\left(\widetilde{\mathcal{A}_{k}}\right), \lambda\left(\widetilde{\mathcal{A}_{k}}\right), \sigma\left(\widetilde{\mathcal{A}_{k}}\right), \delta\left(\widetilde{\mathcal{A}_{k}}\right)\right)$ be the valency of a boundary curve of the amphidrome annulus $\widetilde{\mathcal{A}_{k}}$, and $\mathbf{s}\left(\widetilde{\mathcal{A}_{k}}\right)=-2 \delta\left(\widetilde{\mathcal{A}_{k}}\right) / \lambda\left(\widetilde{\mathcal{A}_{k}}\right)-2 \mathbf{K}\left(\widetilde{\mathcal{A}_{k}}\right)$ be the screw number. Put

$$
\ell\left(\widetilde{\mathcal{A}_{k}}\right)=N /\left\{\lambda\left(\widetilde{\mathcal{A}_{k}}\right) m\left(\widetilde{\mathcal{A}_{k}}\right)\right\}
$$

Note that the above data are independent of the choice of a representative of $\left[B_{i}\right],\left[\mathcal{A}_{j}\right]$ and $\left[\widetilde{\mathcal{A}}_{k}\right]$ respectively. 


\section{THEOREM 1.3.2.}

$$
\begin{aligned}
\operatorname{Lsd}(f, F ; N)= & -\frac{1}{3} \sum_{\left[B_{i}\right]} \sum_{\alpha=1}^{\varphi(i)}\left(\frac{\sigma_{\alpha}^{(i)}+\delta_{\alpha}^{(i)}}{\lambda_{\alpha}^{(i)}}+\sum_{j=1}^{r(\alpha, i)} K_{j}(\alpha, i)\right) \\
& +\sum_{\left[\mathcal{A}_{j}\right]}\left(\frac{\operatorname{gcd}\left(\lambda^{(1)}\left(\mathcal{A}_{j}\right), \lambda^{(2)}\left(\mathcal{A}_{j}\right)\right)}{\ell\left(\mathcal{A}_{j}\right) \lambda^{(1)}\left(\mathcal{A}_{j}\right) \lambda^{(2)}\left(\mathcal{A}_{j}\right)}-\mathbf{K}\left(\mathcal{A}_{j}\right)+\epsilon\left(\mathcal{A}_{j}\right)\right) \\
& +\sum_{\left[\widetilde{\mathcal{A}_{k}}\right]}\left(\frac{1}{2 \ell\left(\widetilde{\mathcal{A}_{k}}\right) \lambda\left(\widetilde{\mathcal{A}_{k}}\right)}-\mathbf{K}\left(\widetilde{\mathcal{A}_{k}}\right)-2\right)
\end{aligned}
$$

where the summations take over all the classes of the orbit decompositions.

Let $\widehat{f}: \widehat{S} \rightarrow \Delta$ be the semi-stable reduction of $f$. The surface $\widehat{S}$ is obtained from $\widetilde{S}$ by the resolution $\widehat{S} \rightarrow \widetilde{S}$ of rational double points of type $A$ at the double points of $\widetilde{F}$. The boundary $\partial \widehat{S}$ of $\widehat{S}$ coincides with $\partial \widetilde{S}$, and has the natural Riemannian metric $h_{\partial \widehat{S}}$. For the fiber germ $(\widehat{f}, \widehat{F})$, we put $\sigma\left(\widehat{f}, \widehat{F} ; h_{\partial \widehat{S}}\right)=\operatorname{Sign}(\widehat{S})+\eta\left(\partial \widehat{S}, h_{\partial \widehat{S}}\right)$. Similarly we define the modified local signature defect of order $N$ as follows, which is also independent of the choice of $h_{\partial S}$.

\section{DEFINITION 1.3.3.}

$$
\operatorname{Lsd}(f, F ; N)=\sigma\left(f, F ; h_{\partial S}\right)-\frac{1}{N} \sigma\left(\widehat{f}, \widehat{F} ; h_{\partial \widehat{S}}\right) .
$$

\section{Corollary 1.3 .4 .}

$$
\begin{aligned}
\operatorname{Lsd}(f, F ; N)= & -\frac{1}{3} \sum_{\left[B_{i}\right]} \sum_{\alpha=1}^{\varphi(i)}\left(\frac{\sigma_{\alpha}^{(i)}+\delta_{\alpha}^{(i)}}{\lambda_{\alpha}^{(i)}}+\sum_{j=1}^{r(\alpha, i)} K_{j}(\alpha, i)\right) \\
& +\sum_{\left[\mathcal{A}_{j}\right]}\left(\frac{\sigma^{(1)}\left(\mathcal{A}_{j}\right)}{\lambda^{(1)}\left(\mathcal{A}_{j}\right)}+\frac{\sigma^{(2)}\left(\mathcal{A}_{j}\right)}{\lambda^{(2)}\left(\mathcal{A}_{j}\right)}+\epsilon\left(\mathcal{A}_{j}\right)\right)+\sum_{\left[\widetilde{\mathcal{A}_{k}}\right]}\left(\frac{\delta\left(\widetilde{\mathcal{A}_{k}}\right)}{\lambda\left(\widetilde{\mathcal{A}_{k}}\right)}-2\right) .
\end{aligned}
$$

REMARK 1.3.5. Tan [34] described the effect of global semi-stable reduction to some global invariants of fibered surfaces in the language of singularity. Therefore Corollary 1.3.4 might be considered as an infinitesimal and also topological version of his results in some sense.

REMARK 1.3.6. The relation between Corollary 1.3.4 and Atiyah's formulation $[7, \S 6]$ for a degeneration of elliptic curves seems to be the following from our viewpoint.

All the monodormies of degenerations of elliptic curves except for a semistable one is periodic, or elliptic element in other words. If we consider the periodic case, our modified local signature defect coincides with the first term of Corollary 1.3.4. This is nothing but the Dedekind sum from our method 
of proof. Our proof is essentially reduced to Dedekind sum by using the orbifold signature theorem, which can be calculated in terms of continued linear fractions (cf. [4]). Since the "moduli" term discussed in $\S 2$ vanishes in this case, this also coincide with our local signature.

On the other hand, "intrinsic local signature" discussed in $[7, \S 6]$ is nothing but the Dedekind sum. This is the reason why our local signature and Atiyah's one coincide with each other for a degenaration of elliptic curves.

We also refer Ogata-Saito [29] for an extension of $[7, \S 6]$ to another direction.

\section{A signature divisor on $\overline{\mathcal{M}}_{g}$}

This section is a summary of a certain part of a joint work with $\mathrm{K}$. Yoshikawa [6]. We define the signature divisor on the Deligne-Mumford compactification. As an explicit signature divisor, we use a certain divisor which comes from Harris-Mumford's formula. As a conclusion, we propose a new type of local signature for Harris-Mumford generic fibration.

2.1. By Deligne-Mumford [10], there exists a coarse moduli space $\overline{\mathcal{M}}_{g}$ of stable curves of genus $g$. Let $\mathcal{M}_{g}$ be the Zariski open subset of $\overline{\mathcal{M}}_{g}$ consisting of the isomorphism classes of smooth stable curves of genus $g$. Then $\mathcal{M}_{g}$ is the coarse moduli space of compact Riemann surfaces of genus $g$. For a stable curve $C$ of genus $g$, the isomorphism class of $C$ is denoted by $[C] \in \overline{\mathcal{M}}_{g}$. For $[C] \in \overline{\mathcal{M}}_{g}$, let $(\operatorname{Def}(C),[C])$ denote the Kuranishi space of $C$ and let $\Gamma_{[C]}:=\operatorname{Aut}(C)$ denote the group of automorphisms of $C$. One has the isomorphisms of germs of complex spaces $(\operatorname{Def}(C),[C]) \cong\left(\operatorname{Ext}^{1}\left(\Omega_{C}^{1}, \mathcal{O}_{C}\right), 0\right)$ and $\left(\overline{\mathcal{M}}_{g},[C]\right) \cong(\operatorname{Def}(C) / \operatorname{Aut}(C),[C]) \cong\left(\operatorname{Ext}^{1}\left(\Omega_{C}^{1}, \mathcal{O}_{C}\right) / \Gamma_{[C]}, 0\right)$ (cf. [10, $\S 1]$ ). Hence $\overline{\mathcal{M}}_{g}$ is a complex orbifold.

There exist irreducible Weil divisors $\Delta_{0}, \ldots, \Delta_{[g / 2]} \subset \overline{\mathcal{M}}_{g}$ such that $\overline{\mathcal{M}}_{g} \backslash$ $\mathcal{M}_{g}=\bigcup_{i=0}^{[g / 2]} \Delta_{i}$. There exists a Zariski open subset $\Delta_{i}^{o} \subset \Delta_{i}$ with the following property: if $[C] \in \Delta_{0}^{o}$, then $C$ is an irreducible stable curve of genus $g$ with a unique node. If $i>0$ and $[C] \in \Delta_{i}^{o}$, then $C$ is a reducible stable curve of genus $g$ with a unique node whose irreducible components consist of a smooth curves of genus $i$ and a smooth curves of genus $g-i$. Following [14, p.51], [15, Cor. 3.95], we define the $\mathbf{Q}$-divisors $\delta_{0}, \ldots, \delta_{[g / 2]}$ and $\delta$ in $\overline{\mathcal{M}}_{g}$ as

$$
\delta_{i}:=\left\{\begin{array}{r}
\Delta_{i}(i \neq 1) \\
\Delta_{1} / 2(i=1)
\end{array}, \quad \delta:=\sum_{i=0}^{[g / 2]} \delta_{i} .\right.
$$

We often identify $\delta_{i}$ and $\delta$ with the corresponding $\mathbf{Q}$-line bundles over $\overline{\mathcal{M}}_{g}$.

Let $S$ be a 2-dimensional complex space with at most rational double point of type A, $B$ is a compact Riemann surface and $f: S \rightarrow B$ is a proper 
flat holomorphic map such that any fiber of $f$ is a stable curve of genus $g$. We call such $f$ a stable fibered surface of genus $g$. Then the induced map $\mu_{f}: B \rightarrow \overline{\mathcal{M}}_{g}$ is defined by

$$
\mu_{f}(b):=\left[f^{-1}(b)\right], \quad b \in B .
$$

Since $S$ is Gorenstein, the dualizing sheaf $\omega_{S}$ of $S$ is locally free. Let $\omega_{S / B}:=$ $\omega_{S} \otimes f^{*} \omega_{B}^{-1}$ denote the relative dualizing sheaf of the family $f$ and set

$$
\lambda(S / B):=\left(\operatorname{det} f_{*} \mathcal{O}_{S}\right) \otimes\left(\operatorname{det} R^{1} f_{*} \mathcal{O}_{S}\right)^{\vee}=\operatorname{det} f_{*} \omega_{S / B},
$$

which is a holomorphic line bundle over $B$. Let $\mathrm{CH}^{1}(B)$ denote the divisor class group of $B$. There exists a unique divisor class $\lambda_{g} \in \operatorname{Pic}\left(\overline{\mathcal{M}}_{g}\right) \otimes \mathbf{Q}$ such that for every stable fibered surface $f: S \rightarrow B$ of genus $g$,

$$
\mu_{f}^{*} \lambda_{g}=\frac{1}{\nu}[\operatorname{div}(s)] \in \mathrm{CH}^{1}(B) \otimes \mathbf{Q}, \quad \forall s \in H^{0}\left(B, \lambda(S / B)^{\otimes \nu}\right), \quad \nu \gg 0 .
$$

The divisor class $\lambda_{g}$ is called the Hodge class.

Inspired by Smith [32], we define the following:

DeFINITION 2.1.1. A Q-divisor $D$ of $\overline{\mathcal{M}}_{g}$ is called a signature divisor if the following identity holds in $\operatorname{Pic}\left(\overline{\mathcal{M}}_{g}\right) \otimes \mathbf{Q}$ :

$$
D \equiv 4 \lambda_{g}-\delta \text {. }
$$

2.2. From now on, by a fibered surface $f: S \rightarrow B$, we mean that $f$ is a proper surjective holomorphic map from a compact connected nonsingular complex surface $S$ to a compact Riemann surface $B$ such that $f$ is relatively minimal, i.e., any fiber of $f$ contains no (-1)-curve. A fiber of $f$ might not be a stable curve in general. The genus of a fibered surface is defined as the genus of its general fiber. Although $f$ is an unstable in general, since $B$ is complex one-dimensional, the induced map from the complement of the critical locus $B \backslash \Sigma_{f}$ to $\mathcal{M}_{g}$ extends to a holomorphic map from $B$ to $\overline{\mathcal{M}}_{g}$ by [18] or by the valuative criterion. This extended map is again written as $\mu_{f}: B \rightarrow \overline{\mathcal{M}}_{g}$ and is called the induced map.

We consider the infinitesimal neighborhood of a fiber of a fibered surface. Let $f_{\text {loc }}:\left(S, S_{0}\right) \rightarrow(\Delta, 0)$ be a relatively minimal one-parameter deformation germ of a curve $S_{0}$ of arithmetic genus $g$, whose total space $S$ is assumed to be smooth. For simplicity, we write $\left(f, S_{0}\right)=\left(\left(f_{\mathrm{loc}}, S_{0}\right)\right)$ for $f_{\mathrm{loc}}:\left(S, S_{0}\right) \rightarrow$ $(\Delta, 0)$ and consider it as a fiber germ. We often identify a fiber germ with its representative.

Definition 2.2.1. Let $\mathcal{A}$ be a subset of $\mathcal{M}_{g}$. A fiber germ $f:\left(S, S_{0}\right) \rightarrow$ $(\Delta, 0)$ of genus $g$ is said to be $\mathcal{A}$-general if the induced map satisfies $\mu_{f}(\Delta \backslash$ $\{0\}) \subset \mathcal{A}$. A fibered surface $f: S \rightarrow B$ of genus $g$ is $\mathcal{A}$-general if the fiber germ $f:\left(S, S_{b}\right) \rightarrow(B, b)$ is $\mathcal{A}$-general for all $b \in B$. The set of all $\mathcal{A}$-general fiber germs of genus $g$ is denoted by $\operatorname{Germ}(\mathcal{A})$. 
Definition 2.2.2. Let $\mathcal{A}$ be a subset of $\mathcal{M}_{g}$. A function $\sigma_{\mathcal{A}}: \operatorname{Germ}(\mathcal{A})$ $\rightarrow \mathbf{Q}$ is called a local signature with respect to $\mathcal{A}$ if the following hold:

(1) If $\left[S_{0}\right] \in \mathcal{A}$ and if $f:\left(S, S_{0}\right) \rightarrow(\Delta, 0)$ is an $\mathcal{A}$-general fiber germ, then

$$
\sigma_{\mathcal{A}}\left(f, S_{0}\right)=0 \text {. }
$$

(2) For every $\mathcal{A}$-general fibered surface $f: S \rightarrow B$ of genus $g$,

$$
\operatorname{Sign}(S)=\sum_{b \in B} \sigma_{\mathcal{A}}\left(f, S_{b}\right),
$$

which is a finite sum by (1).

Once a signature divisor $\mathcal{D} \subset \overline{\mathcal{M}}_{g}$ is given, we can associate the corresponding local signature with respect to $\mathcal{M}_{g} \backslash \operatorname{Supp} \mathcal{D}$ as follows.

Let $\widehat{f}_{\text {loc }}: \widehat{S} \rightarrow \Delta$ be the normally minimal model of the relatively minimal fibration $f_{\text {loc }}: S \rightarrow \Delta$, and let $\# \mathrm{Bd}_{N R}$ is the number of contractions of $(-1)$-curves to obtain $f_{\text {loc }}$ starting from $\widehat{f}_{\text {loc }}$. Let $\Delta^{\prime} \rightarrow \Delta$ be the cyclic cover totally ramified at the origin whose order coincides with the minimal pseudo-period $N_{0}$ of the monodormy map of $f_{\text {loc }}$, and let $f^{\prime}: S^{\prime} \rightarrow \Delta^{\prime}$ be the semi-stable reduction of $f_{\text {loc }}$. Let $\mu_{f^{\prime}}: \Delta^{\prime} \rightarrow \bar{M}_{g}$ be the induced map. By using the modified local signature defect of order $N_{0}$, we define

$$
\widehat{\operatorname{Lsd}}\left(f, S_{0}\right):=\operatorname{Lsd}\left(f, S_{0} ; N_{0}\right)+\# \mathrm{Bd}_{N R} .
$$

This is an invariant of the fiber germ $\left(f_{\text {loc }}, S_{0}\right)$.

TheOREM 2.2.3. Let $\mathcal{D}_{\text {sign }}$ be a signature divisor on $\overline{\mathcal{M}}_{g}$. Then

$$
\sigma_{\mathcal{A}}\left(f, S_{0}\right):=\frac{1}{N_{0}} \operatorname{mult}_{t=0}\left[\mu_{f}^{*} \mathcal{D}_{\text {sign }}\right]+\widehat{\operatorname{Lsd}}\left(f, S_{0}\right)
$$

is a local signature with respect to $\mathcal{A}=\mathcal{M}_{g} \backslash \operatorname{Supp}\left(\mathcal{D}_{\text {sign }}\right)$, where mult $_{t=0}\left[\mu_{f}^{*} \mathcal{D}_{\text {sign }}\right]$ is the multiplicity of the divisor $\mu_{f^{\prime}}^{*} \mathcal{D}_{\text {sign }}$ at the origin of $\Delta^{\prime}$.

2.3. For a compact Riemann surface $C$, let $\mathbf{C}(C)$ denote the field of meromorphic functions on $C$. The gonality of $C$ is the integer defined by

$$
\operatorname{gon}(C):=\min \{\operatorname{deg} f ; f \in \mathbf{C}(C), f \text { is not constant }\} .
$$

Then $\operatorname{gon}(C) \leq[(g(C)+3) / 2]$. When $g=2 k-1$, define the subset $\mathcal{D}_{\mathrm{HM}}$ of $\mathcal{M}_{g}$ by

$$
\mathcal{D}_{\mathrm{HM}}:=\left\{[C] \in \mathcal{M}_{g} ; \operatorname{gon}(C) \leq k=(g+1) / 2\right\} \subset \mathcal{M}_{g} .
$$

Then we define the Harris-Mumford divisor of $\overline{\mathcal{M}}_{g}$ as the closure $\overline{\mathcal{D}}_{\mathrm{HM}}$ of $\mathcal{D}_{\mathrm{HM}}$ in $\overline{\mathcal{M}}_{g}$, which is a Weil divisor. 
Theorem 2.3.1. (Harris-Mumford [14]) When $g=2 k-1$, the following identity holds in $\operatorname{Pic}\left(\overline{\mathcal{M}}_{g}\right) \otimes \mathbf{Q}$ :

$$
\overline{\mathcal{D}}_{\mathrm{HM}} \equiv \frac{(2 k-4) !}{k !(k-2) !}\left\{6(k+1) \lambda_{g}-k \delta_{0}-\sum_{i=1}^{k-1} 3 i(2 k-1-i) \delta_{i}\right\} .
$$

In particular, the following $\mathbf{Q}$-divisor is a signature divisor of $\overline{\mathcal{M}}_{g}$ when $g=2 k-1$ :

$$
\begin{aligned}
\mathcal{D}_{\text {signHM }}:= & \frac{2 \cdot k !(k-2) !}{3(k+1)(2 k-4) !} \overline{\mathcal{D}}_{\mathrm{HM}}-\frac{k+3}{3(k+1)} \delta_{0} \\
& +\sum_{i=1}^{k-1} \frac{2 i(2 k-1-i)-(k+1)}{k+1} \delta_{i} .
\end{aligned}
$$

Definition 2.3.2. The Harris-Mumford local signature is defined as

$$
\begin{aligned}
\sigma_{H M}\left(f, S_{b}\right)= & \frac{1}{N_{b}} \cdot \operatorname{mult}_{b^{\prime}}\left[\mu_{f^{\prime}}^{*} \mathcal{D}_{\text {signHM }}\right]+\widehat{\operatorname{Lsd}}\left(f, S_{b}\right) \\
= & \frac{1}{N_{b}}\left\{\frac{2 \cdot k !(k-2) !}{3(k+1)(2 k-4) !} \operatorname{mult}_{b^{\prime}}\left[\mu_{f^{\prime}}^{*} \overline{\mathcal{D}}_{\mathrm{HM}}\right]-\frac{k+3}{3(k+1)} \operatorname{mult}_{b^{\prime}}\left[\mu_{f^{\prime}}^{*} \delta_{0}\right]\right. \\
& \left.+\sum_{i=1}^{k-1} \frac{2 i(2 k-1-i)-(k+1)}{k+1} \operatorname{mult}_{b^{\prime}}\left[\mu_{f^{\prime}}^{*} \delta_{i}\right]\right\}+\widehat{\operatorname{Lsd}}\left(f, S_{b}\right),
\end{aligned}
$$

where $N_{b}$ is the minimal pseudo-period of the monodromy map around $S_{b}$.

When $\mathcal{A}=\mathcal{M}_{g} \backslash \operatorname{Supp} \mathcal{D}_{\mathrm{HM}}$ in the Definition 2.2.1, an $\mathcal{A}$-general fibered surface of genus $g$ is said to be Harris-Mumford general.

Theorem 2.3.3. Let $f: S \rightarrow B$ be a Harris-Mumford general fibered surface. Then

$$
\text { Sign } S=\sum_{b \in B} \sigma_{H M}\left(f, S_{b}\right) \text {. }
$$

Corollary 2.3.4. Let $f: S \rightarrow B$ be a non-hyperelliptic fibered surface of genus 3 . Then

$$
\begin{aligned}
\operatorname{Sign} S=\sum_{b \in B} \sigma_{H M}\left(f, S_{b}\right)= & \sum_{b \in B}\left\{\frac { 1 } { N _ { b } } \left(\frac{4}{9} \text { mult }_{b^{\prime}}\left[\mu_{f^{\prime}}^{*} \overline{\mathcal{D}}_{\text {hyper }}\right]-\frac{5}{9} \text { mult }_{b^{\prime}}\left[\mu_{f^{\prime}}^{*} \delta_{0^{\prime}}\right]\right.\right. \\
& \left.\left.+\frac{1}{3} \operatorname{mult}_{b^{\prime}}\left[\mu_{f^{\prime}}^{*} \delta_{1}\right]\right)+\widehat{\operatorname{Lsd}}\left(f, S_{b}\right)\right\}
\end{aligned}
$$

where $\overline{\mathcal{D}}_{\text {hyper }}$ is the divisor defined as the closure of the hyperelliptic locus in $\overline{\mathcal{M}}_{3}$.

EXAMPLE 2.3.5. If $\left(f, S_{b}\right)$ is a germ of a generic smooth hyperelliptic curve (resp. a generic non-separated Lefschetz fiber, resp. a generic separated Lefschetz fiber) in a non-hyperelliptic fibered surface of genus 3 , then 
$\sigma_{H M}\left(f, S_{b}\right)=4 / 9($ resp. $-5 / 9$, resp. $1 / 3)$. Therefore $\sigma_{H M}$ coincide with Kuno's local signature [21] in these cases.

\section{References}

[1] T. Arakawa, T. Ashikaga, Local splitting families of hyperelliptic pencils, I: Tohoku Math. J. 53 (2001), 369-394. II: Nagoya Math. J. 175 (2004), 103-124.

[2] T. Ashikaga, Local signature defect of fibered complex surfaces via monodromy and stable reduction, Comment. Math. Helv. 85 (2010), 417-461.

[3] T. Ashikaga, H. Endo, Various aspects of degenerate families of Riemann surfaces, Sugaku Exposition 19 (2006), Amer. Math. Soc., 171-196.

[4] T. Ashikaga, M. Ishizaka, A geometric proof of the reciprocity law of Dedekind sum, Preprint (2009).

[5] T. Ashikaga, K. Konno, Global and local properties of pencils of algebraic curves, in Algebraic Geometry 2000 Azumino, ed. by S. Usui et al., Adv. Stud. Pure Math. 36 (2002), 1-49.

[6] T. Ashikaga, K. Yoshikawa, $A$ divisor on the moduli space of curves associated to the signature of fibered surfaces (with an appendix by K. Konno), Adv. Stud. Pure Math. 56, Math. Soc. Japan, Tokyo 2009, 1-34.

[7] M. Atiyah, The logarithm of Dedekind $\eta$-function, Math. Ann. 278 (1987), 335-380.

[8] M. Atiyah, V. K. Patodi, I. M. Singer, Spectral asymmetry and Riemannian geometry, I, Math. Proc. Cambridge Philos. Soc. 77 (1975), 43-69, II, ibid. 78 (1975), 405-432.

[9] Z. Chen, S. L. Tan, Upper bound on the slope of a genus 3 fibration, Preprint, (2005).

[10] P. Deligne, D. Mumford, The irreducibility of the space of curves of given genus, Publ. Math. I.H.E.S. 36 (1969), 75-110.

[11] H. Endo, Meyer's signature cocycle and hyperelliptic fibrations (with Appendix written by T. Terasoma), Math. Ann. 316 (2000), 237-257.

[12] M. Furuta, Surface bundle and local signature, in; The report of the symposium of Topology related to Riemann surfaces, ed. by N. Kawazumi, pp. 47-53, 1999, in Japanese.

[13] M. Furuta, Surface bundle and local signature, in; The report of the symposium of Algebraic Geometry at Kinosaki, pp. 94-103, 2000, in Japanese.

[14] J. Harris, D. Mumford, On the Kodaira dimension of the moduli space of curves, Invent. Math. 67 (1982), 23-86.

[15] J. Harris, I. Morrison, Moduli of Curves, Springer, Berlin, (1998).

[16] E. Horikawa, On algebraic surfaces with pencils of curves of genus 2, In Complex Analysis and Algebraic Geometry, a volume dedicated to K. Kodaira, Iwanami Shoten Publishers and Cambridge University Press, Tokyo and Cambridge, (1977), 79-90.

[17] S. Iida, Adiabatic limits of $\eta$-invariants and the Meyer functions, Math. Ann. 346 (2010), 669-717.

[18] Y. Imayoshi, Holomorphic families of Riemann surfaces and Teichmüller spaces, In Riemann surfaces and related topics, Ann. of Math. Stud. 97, Princeton Univ. Press, (1981), 277-300.

[19] K. Konno, Clifford index and the slope of fibered surfaces, J. Algebraic Geom. 8 (1999), 207-220.

[20] K. Konno, An appendix to the paper of Ashikaga and Yoshikawa (6), Preprint (2008).

[21] Y. Kuno, The mapping class group and the Meyer function for plane curves, Math. Ann. 342 (2008), 923-949. 
[22] Y. Matsumoto, J. M. Montesinos-Amilibia, Pseudo-periodic maps and degeneration of Riemann surfaces, I, II, Preprints, Univ. of Tokyo and Univ. Complutense de Madrid, 1991/1994 (Revised in 2006).

[23] Y. Matsumoto, J. M. Montesinos-Amilibia, Pseudo-periodic maps and degeneration of Riemann surfaces, Bull. Amer. Math. Soc. 30 (1994), 70-75.

[24] Y. Matsumoto, On 4-manifolds fibered by tori, I, Proc. Japan Acad. 58 (1982), 298301; II, ibid. 59 (1983), 100-103.

[25] Y. Matsumoto, Lefschetz fibrations of genus two - a topological approach, in Proceedings of the 37th Taniguchi Symposium on "Topology and Teichmüller Spaces", World Scientific, Singapore, (1996), 123-148.

[26] T.Morifuji, On Meyer's function of hyperelliptic mapping class groups, J. Math. Soc. Japan 55 (2003), 117-129.

[27] J. Nielsen, Die Structur periodischer Transformation von Flächen, Mat. -Fys. Medd. Danske Vid. Selsk. 15 (1937). English translation : in Collected Papers 2, Birkhäuser, 1986.

[28] J. Nielsen, Surface transformation classes of algebraically finite type, Mat.-Fys.Medd. Danske Vid. Selsk. 21 (1944), 3-89. English translation; in Collected Papers 2, Birkhäuser, 1986.

[29] S. Ogata, M. Saito, Signature defect and eta functions of degenerations of Abelian varieties, Japan. J. Math. 23, 319-364.

[30] M. Reid, Problems on pencils of small genus, Preprint (in his home page), (1990).

[31] M. Sato, Local signature for fibrations with a finite group action, Preprint (arXiv: 0912.1952v1 [math.GT]10Dec2009).

[32] I. Smith, Lefschetz fibrations and the Hodge bundle, Geometry and Topology 3 (1999), 211-233.

[33] S. Takamura, Towards the classification of atoms of degenerations II, (Cyclic quotient construction of degenerations of complex curves), RIMS Preprint 1344 (2001).

[34] S. L. Tan, On the invariants of base changes of pencils of curves, I: Manuscripta Math. 84 (1994), 225-244. II: Math. Z. 222 (1996), 655-676.

[35] K. Ueno, Discriminants of curves of genus 2 and arithmetic surfaces, in Algebraic Geometry and Commutative Algebra in honor of M. Nagata, Kinokuniya, Tokyo, (1987), 749-770.

[36] G. Xiao, The fibrations of algebraic surfaces, Shanghai Scientific and Technical Publishers, (1992), in Chinese.

[37] K. Yoshikawa, A local signature for generic 1-parameter deformation germs of a complexcurve, in The report on the symposium on Algebraic Geometry and Topology of Degenerations, Coverings and Singularities (2000), 188-200, in Japanese.

FACULTY OF ENGINEERING

TOHOKU-GAKUIN UNIVERSITY,

TAGAJO, MIYAGI, 985-8537, JAPAN

E-mail: tashikaga@tjcc.tohoku-gakuin.ac.jp

Received November 28, 2009. 